\section{RMD Open}

Rheumatic \&

Musculoskeletal Diseases

To cite: Favero $\mathrm{M}$, Ramonda R, Goldring MB, et al. Early knee osteoarthritis. RMD Open 2015;1:e000062. doi:10.1136/rmdopen-2015000062

- Prepublication history and additional material for this paper is available online. To view these files please visit the journal online (http://dx.doi.org/10.1136/ rmdopen-2015-000062)

Received 3 February 2015 Revised 30 May 2015 Accepted 14 June 2015

\section{(1) cossnath}

${ }^{1}$ Rheumatology Unit, Department of MedicineDIMED, University Hospital of Padova, Padova, Italy

${ }^{2}$ Laboratory of Immunorheumatology and Tissue Regeneration/ RAMSES, Rizzoli Orthopedic Research Institute, Bologna, Italy

${ }^{3}$ Research Division, Hospital for Special Surgery and Weill Cornell Medical College, New York, New York, USA

Correspondence to Dr Marta Favero; faveromarta@gmail.com

\title{
Early knee osteoarthritis
}

\author{
Marta Favero, ${ }^{1,2}$ Roberta Ramonda, ${ }^{1}$ Mary B Goldring, ${ }^{3}$ Steven R Goldring, ${ }^{3}$ \\ Leonardo Punzi ${ }^{1}$
}

\section{ABSTRACT}

Concepts regarding osteoarthritis, the most common joint disease, have dramatically changed in the past decade thanks to the development of new imaging techniques and the widespread use of arthroscopy that permits direct visualisation of intra-articular tissues and structure. MRI and ultrasound allow the early detection of pre-radiographic structural changes not only in the peri-articular bone but also in the cartilage, menisci, synovial membrane, ligaments and fat pad. The significance of MRI findings such as cartilage defects, bone marrow lesions, synovial inflammation/effusions and meniscal tears in patients without radiographic signs of osteoarthritis is not fully understood.

Nevertheless, early joint tissue changes are associated with symptoms and, in some cases, with progression of disease. In this short review, we discuss the emerging concept of early osteoarthritis localised to the knee based on recently updated knowledge. We highlight the need for a new definition of early osteoarthritis that will permit the identification of patients at high risk of osteoarthritis progression and to initiate early treatment interventions.

\section{INTRODUCTION}

Osteoarthritis (OA) is the most common joint disease, causing disability and reduction of quality of life and participation in social activity. Now considered a whole joint disease, OA is characterised by cartilage loss, subchondral bone changes, synovial inflammation and meniscus degeneration. ${ }^{1}$ Many studies have focused on understanding the pathophysiological mechanisms of $\mathrm{OA}$ in humans by histological examination of retrieved joint tissues from patients with OA or by employing cells, including chondrocytes or synoviocytes, isolated from tissues obtained from patients undergoing joint replacement, or by studying whole joint tissue in explant cultures. ${ }^{2}$ In vitro research approaches such as these permit the study of the late stage of OA, but the biological processes involved in the initiation and in the early stages of joint disease in any given human joint are not well understood. In addition, there is no therapy shown consistently to delay the progression of OA disease. The limited efficacy of non-surgical

\section{Key messages}

Concepts regarding osteoarthritis have dramatic ally changed in the past decade thanks to the development of new imaging techniques. MRI and ultrasound allow the early detection of preradiographic structural changes not only in the peri-articular bone but also in the cartilage, menisci, synovial membrane, ligaments and fat pad.

- In this short review, we discuss the emerging concept of early osteoarthritis localised to the knee based on recently updated knowledge.

- We highlight the need for a new definition of early osteoarthritis that will permit the identification of patients at high risk of osteoarthritis progression and to initiate early treatment interventions.

treatments for OA may be due partly to their use at a late point in the evolution of disease when structural deterioration is often advanced. ${ }^{3}$ Concerning animal models, it has been demonstrated that gene expression in joint tissues is different in younger mice as compared with older mice. ${ }^{4}$ For example, a 10-week-old C57BL/6 mouse corresponds approximately to an adolescent human, while a 12-month-old mouse represents a human aged $40-50$ years. $^{5}$ These data highlight the importance of considering the ages of animals used in models of OA.

Recently, national and international scientific societies have demonstrated great interest in 'early osteoarthritis' (EOA), as happened several years ago for rheumatoid arthritis. Even though several study groups of experts in the OA field have been created, a definition of EOA is still lacking. It is important to identify patients affected by EOA in order to initiate early interventions and therapeutic approaches that could prevent progression and severe structural changes in the joint associated with later stages of OA. The main objective of the present short nonsystematic narrative review is to highlight recent insights from the Osteorheumatology 
Meeting held in Genoa in October 2014. The concept of EOA localised to the knee will be discussed by presenting the updated knowledge in this field. Old and new concepts of EOA will be explored according to different points of view. Many aspects can be considered in classifying OA as early, including the pathological processes, age of onset, symptoms, histology and imaging.

\section{PATHOPHYSIOLOGICAL MECHANISIMS IN EARLY KNEE OA}

Mechanical injury, hereditary factors and ageing can initiate the pathophysiological processes that lead to OA. At the beginning, a hypertrophic repair phase can occur, resulting in softening of the articular cartilage due to increased water content secondary to glycosaminoglycan (GAG) loss. In this phase, anabolic activities and production of collagen type II and proteoglycan are actually increased. Chondrocytes appear in clusters as the result of their increased proliferation rate. ${ }^{6}{ }^{7}$ Cartilage mechanical properties are sensitive to composition and structure, so the loss of GAG content occurring in the early phase of the disease leads to alteration in cartilage compressive resistance and osmotic pressure within the tissue. 8

Subsequently, in the early phase of the disease, the amplification of catabolic activity occurs, associated with increased expression of inflammatory mediators, cartilage-degrading proteinases and stress response factors. These alterations lead to cartilage loss, from fibrillation of the superficial zone to more complex and deeper fissures. Collagen type II fragments from the damaged cartilage surface can induce inflammatory responses in the synovial membrane resulting in hyperplasia, lymphocytic infiltration and perivascular lymphoid aggregates. ${ }^{9}$ Inflammatory mediators released by inflamed synovium can promote cartilage degradation. ${ }^{10}$

EOA can be defined histologically as having a grade of $1.0-3.0$ by the OARSI scoring system, which takes into account the depth of degradation into articular cartilage. ${ }^{71}$ Grade 1.0 is characterised by swelling of the articular cartilage, but mild fibrillation in the superficial zone can be present. In grade 2.0, small regions of the cartilage surface are lost, cracks extend deeper into the superficial zone, and chondrocytes have begun to form clusters. In grade 3.0, vertical fissures have advanced into the middle zone and formation of chondrons is seen. EOA changes affect only the superficial and the middle zones of the cartilage. ${ }^{11}$

\section{RISK FACTORS OF EARLY KNEE OA}

There is ample evidence that anterior cruciate ligament (ACL) rupture and meniscal tear are two major risks factors for developing EOA. ${ }^{12}$ Injuries to the ACL frequently occur in young patients, especially in the athletes, leading to pain, functional impairment and reduced physical activity in the young or middle-aged adult, the so-called young patients with old knees. ACL rupture is a complex event and concomitant meniscal injury, collateral ligament tear, cartilage damage and bone contusion can be detected as a consequence of trauma. ${ }^{13} 14$ There are an estimated 900000 cases of knee injuries annually in the USA and post-traumatic OA accounts for $12 \%$ of all cases of OA. ${ }^{15}$ The prevalence of reported OA after ACL injury ranges between $10 \%$ and $90 \%$ according to different studies. ${ }^{16}$ Also, untreated meniscal damage is a known risk factor for developing OA with an OR of 5.7 during 30 months of follow-up. ${ }^{17}$ Similarly, a partial meniscectomy increases the risk of $\mathrm{OA}$ by four fold, as assessed at 16 years of post-surgery. ${ }^{18}$

EOA changes have been attributed in part to the increased joint instability and altered joint mechanics that result from ACL disruption or meniscal tear. In this environment, the articular cartilage is exposed to abnormal biomechanical forces and it has been suggested that these influences are responsible for deregulated chondrocyte matrix synthesis and repair capacity. Such acute mechanical injuries also result in the release of biological mediators such as cytokines, proteolytic enzymes and reactive oxygen species that may adversely affect chondrocyte survival and synthetic activity. ${ }^{19} 20$

Several studies have found increased concentrations of cytokines in synovial fluid aspirated from the injured knees of patients with acute, subacute and chronic ACL deficiency. ${ }^{21} 22$ The role of inflammation in the initiation of OA following meniscal damage has also been highlighted recently. ${ }^{23}$ In support of this concept, in patients with traumatic meniscal injury but no radiographic evidence of $\mathrm{OA}$, the synovium retrieved during meniscectomy is frequently inflamed and inflammation scores are associated with increased pain and dysfunction and a unique chemokine profile. ${ }^{24}$ The presence of additional OA risk factors in patients with ACL rupture or meniscal tear, such as obesity, age, joint malalignment or genetic risk factors, may lead to more unfavourable outcomes. Moreover, some new or controversial risk factors have been associated with early knee structural changes detected by magnetic resonance imaging (MRI), including smoking, physical inactivity, muscle weakness, leptin, vitamin D deficiency and dietary fatty acid intake. ${ }^{25}$

To identify people at high risk of developing OA, it is important to initiate early interventional treatment in patients with early structural changes, even if they are asymptomatic. Since it is well established that ACL rupture and meniscal tear leads to $\mathrm{OA}$, injured patients represent a unique opportunity to study and understand the pathological mechanisms involved in the early phase of the disease. Potent anti-inflammatory drugs have been administered at an early stage after ACL tear to test their potential to prevent the development of the disease. ${ }^{26}$

\section{SYMPTOMS IN EOA}

The hallmark symptom of knee OA is pain and two different patterns of pain have been described according to the disease stage. In the early phases of the disease, 
pain is related to activity and becomes more constant over time, while in the late stages there is 'background pain' interspersed with unpredictable intense pain. ${ }^{27}$ Radiographic evidence of OA is a relatively late marker for the structural evolution of the disease. Large epidemiological studies have demonstrated that X-ray abnormalities often occur in the absence of pain. The prevalence of asymptomatic knee OA among adults aged 45 >years was $12 \%$ and $11 \%$, respectively, in the Framingham study and Johnston County Osteoarthritis Project. $^{28} 29$ One possible reason is that pain-sensitive features in the joint are not visualised by the radiographs. Early osteochondral defects and meniscal tears detected by MRI have been found incidentally in middle-aged and elderly people and are often not associated with knee pain or other symptoms. ${ }^{30}$ This finding highlights the concept that EOA changes can be asymptomatic and pain, stiffness and aching may occur later in the history of the disease when the OA pathological process is far advanced. ${ }^{3}$

Pain is a very complex phenomenon and it is important to understand why it occurs with precise timing in the joint structures that are responsible for producing it inside the knee because new therapies can be specifically targeted. In a recent systematic review of the literature, bone marrow lesions (BMLs) and effusion/ synovitis have been correlated with pain in knee OA, suggesting that these features may indicate the origin of pain in knee OA. ${ }^{31}$

\section{CLASSIFICATION OF EARLY KNEE OA: NEED FOR NEW CRITERIA FOR THE DIAGNOSIS}

Both the American College of Rheumatology (ACR) criteria of $1986^{32}$ and the recent European League against Rheumatism (EULAR) recommendations in $2010^{33}$ do not provide criteria for the diagnosis of EOA. The ACR criteria are based on symptoms, especially pain; age more than 50; clinical signs; radiographs and laboratory findings, the latest in order to rule out autoimmune or inflammatory arthritis.

It is surprising that the 2010 EULAR recommendations $^{33}$ do not significantly differ from the 1986 ACR criteria: OA is defined as a common joint disorder showing focal cartilage loss, new bone formation and involvement of all joint tissues, introducing the new concept of OA as a whole joint disease; however, the diagnosis is still based on symptoms (usage-related pain, shortlived stiffness), functional limitation, clinical and radiographic findings, and risk factors (age, gender, body mass index (BMI), occupation, family history of OA, history of knee injury, etc). Nevertheless, in the future research agenda, EULAR highlights the importance of developing diagnostic criteria for early symptomatic knee OA.

Recently, Luyten et $a l^{34}$ have proposed classification criteria for early knee OA, with the aim of identifying a subpopulation of patients with signs of emerging joint disease and potentially at a higher risk to develop frank
$\mathrm{OA}$ of the knee. The authors, experts in treatment of cartilage lesions of the knee, underlined the need for better characterisation of the patients for inclusion in regenerative medicine clinical trials. The classification is based on symptoms of EOA (pain: at least two episodes for 10 days in the past year), structural changes as defined by regular radiographs (Kellgren-Lawrence (K-L) grade 0 or 1 or 2 osteophytes only) and early degenerative changes detected by new imaging techniques and arthroscopy. ${ }^{34}$

\section{IMAGING TECHNIQUES IN EARLY KNEE OA: FROM RADIOGRAPHS TO MRI}

Plain radiography is considered the current 'gold standard' for morphological assessment of knee OA, while other imaging modalities such as MRI and sonography are seldom indicated for the diagnosis of OA. ${ }^{33}$ Bone sclerosis, subchondral cysts, osteophytes and joint space narrowing, an indirect sign of cartilage loss, can be detected by radiographs.

The K-L scale, which takes into account several radiographic features of OA, including joint space narrowing and osteophyte development, has been traditionally exploited since 1957 to assess the severity of radiographic knee OA and is still used in clinical trials to assess disease-modifying OA drugs. ${ }^{35}$ The explanation is feasibility and tradition, as well as the fact that no clear cut-off or overall severity grade exists for OA in the MRI classification criteria. ${ }^{36}$

The K-L grading scale usually defines the presence or absence of OA using grade 2 as the threshold, or according to a recently proposed new definition, grade 2/ osteophytes (osteophytes only, no joint space narrowing) ${ }^{37}$ In many studies, the K-L grades 1 and 2 have been considered as evidence of early radiographic OA. Five different descriptions of the K-L score have been found in the literature resulting in disagreement in defining and grading OA between different study cohorts. ${ }^{38}$ Different versions of the K-L criteria have impacted the classification of $\mathrm{OA}$, especially in the lowest grade $(\mathrm{K}-\mathrm{L} \geq 1)$, in which all alternative $\mathrm{K}-\mathrm{L}$ descriptions classified more knees as having OA than the original classification. ${ }^{39}$ Schiphof et al found that the reproducibility of two trained readers was low for the cut-off $\mathrm{K}-\mathrm{L} \geq 1$, due probably to the influence of personal interpretation of possible osteophytic lipping. The authors suggested the use of alternative K-L versions instead of the original one to distinguish no OA $(\mathrm{K}-\mathrm{L}=0)$ from possible OA $(\mathrm{K}-\mathrm{L}=1) .{ }^{39}$ Nevertheless, the same group have has recently demonstrated the achievement of a good interobserver reliability between trained readers and an experienced reader for the assessment of EOA grading. ${ }^{40}$ The differences between these studies may be due to the type of training received by the readers for the assessment of EOA grading.

Nevertheless, by the time the first knee joint changes are detected by radiography, more than $10 \%$ of the 
cartilage is already lost. ${ }^{41}$ There are many joint tissues not visible by radiographs, including the cartilage, synovium, meniscus, ligaments, capsule and fat pad. The concept of EOA is dramatically changed since the introduction of new imaging techniques such as MRI, which is more sensitive for detecting early structural changes, not only in the bone, but also in all joint tissues. MRI enables visualisation of knee $\mathrm{OA}$ on a spectrum from a normal joint through EOA to clinically and radiographically evident $\mathrm{OA},{ }^{41}$ detecting cartilage defects, loss of cartilage volume, subchondral bone changes, BMLs, synovitis and meniscal tears. ${ }^{42}$

Two semiquantitative scoring methods have been developed for assessing joint changes detected by MRI in knee OA: the Whole Organ Magnetic Resonance imaging Score (WORMS ${ }^{43}$ and the Boston Leeds Osteoarthritis Knee Score (BLOKS). ${ }^{44}$ Both of these scores are used in the classification criteria for EOA proposed by Luyten for grading cartilage morphology, meniscal tears and BMLs. ${ }^{34}$

New methodological approaches for compositional quantitative assessment have been introduced and MRI techniques such as T2 mapping, dGEMRIC and T1rho are currently used in clinical trials focused on EOA to evaluate collagen or GAG content in the cartilage and meniscus. ${ }^{45}$ Many studies have evaluated the significance of MRI lesions predominantly in knees with prevalent radiographic OA (K-L grade $\geq 2$ ). ${ }^{31}{ }^{46}$ Nevertheless, early structural changes detected by MRI are frequently also observed in community-based studies of younger individuals and/or people without diagnosed OA according to the ACR criteria or significant symptoms requiring intervention. ${ }^{25}$ Cartilage defects are detected by MRI at three times greater prevalence than by radiography before the age of 45 years and are commonly observed in younger and/or predominantly 'healthy' non-OA cohorts with a prevalence of up to $71 \%$ in healthy women. ${ }^{25}{ }^{47}$ Cartilage defects in a number of younger community-based populations have been shown to decrease over time. ${ }^{48} 49$

This suggests that cartilage lesions may be reversible, especially in populations with younger age, lower BMI, decreased BMI, lower cartilage volume, smaller tibial bone size and lack of radiographic change. ${ }^{47}$

Even BMLs and meniscal tears are visualised by MRI in 'healthy' individuals or patients with EOA at a prevalence of up to $39 \%$ and $72 \%$, respectively. ${ }^{30} 50$

BMLs have been detected at a lower rate in healthy participants than in OA patients. Incident BLMs were associated with BMI and development of pain. Moreover, BMLs have been demonstrated to predict increased knee cartilage defects and loss of cartilage volume in healthy middle-age adults without knee pain over 2 years. ${ }^{51}$

Nevertheless, BMLs may be reversible and can decrease in size and even resolve in younger and/or healthy participants. ${ }^{52}$ It is important to study pre-radiographic or 'relatively healthy' populations with early MRI structural changes in order to understand OA development and to identify risk factors that will permit identifying early strategies of intervention for addressing cartilage defects and BML. In a cohort of 855 patients (mean age 31 years) with subacute knee symptoms (pain more than 4 weeks) and K-L grades 0 and 1 , the development of OA at 10 years was associated with ACL and meniscal lesions visualised by MRI at the baseline. The incidence of OA $(\mathrm{K}-\mathrm{L} \geq 2)$ was $23 \%$ for tibiofemoral $\mathrm{OA}$ and $22 \%$ for patellofemoral OA. ${ }^{53}$

MRI lesions have been evaluated recently by Sharma et $a \tilde{l}^{4}$ in a cohort of 849 Osteoarthritis Initiative participants at increased risk for OA but with K-L grade 0 in both knees. Cartilage defects were found by MRI in $75.5 \%$ of patients, BMLs in $60.5 \%$, meniscal tears in $21.2 \%$ and meniscal extrusions in $13.9 \%$. Interestingly, cartilage damage, BMLs and meniscal extrusion correlated with prevalent and frequent symptoms at the baseline. Moreover, cartilage damage, BMLs and meniscal lesions were each associated significantly with the incidence of persistent symptoms at 60 months of follow-up. ${ }^{54}$ BMLs were associated with incident patellofemoral cartilage damage. The frequencies of joint lesions found in this study were consistent with those described in the literature in patients without radiographic signs of OA. ${ }^{30} 5055-57$ The authors concluded that pre-radiographic MRI lesions, as suggested by other studies, ${ }^{57}$ were not an incidental finding, since they were associated with the prevalence and incidence of symptoms in the population of patients at increased risk of OA. A limitation of this study was that the MRI reading did not include effusion and synovitis, which can correlate with the onset of the symptoms. Synovitis is common in EOA and late OA and is associated with OA symptoms and progression. ${ }^{58} 59$

Synovial inflammation is a factor that contributes to dysregulation of chondrocyte function, favouring an imbalance between the catabolic and anabolic activities of chondrocytes in remodelling the cartilage extracellular matrix. ${ }^{60}$ Synovial inflammation evaluated as lymphocytic infiltration by histology has been found to correlate with symptoms in patients without radiographic evidence of OA $(\mathrm{K}-\mathrm{L}=0)$ undergoing meniscal surgery for meniscal tear. ${ }^{24}$ Moreover, baseline effusion and/or Hoffa synovitis detected by MRI in patients with nonradiographic signs of $\mathrm{OA}$ have been correlated with the development of OA in two recent studies. ${ }^{61} 62$

Radiographically invisible pathologies such as cartilage defects and meniscal tears can also be seen by arthroscopy. ${ }^{63}$ Arthroscopy, with its tactile and dynamic capabilities, permits palpation of the joint tissues with a probe, allowing detection of softening, which is the earliest change in the cartilage. The Outerbridge $\operatorname{score}^{64}$ was developed for the assessment of cartilage changes; a grading of I (softening) and II (cartilage surface damage $<50 \%$ of the tissue depth) can be considered as signs of EOA.

Even ultrasonography (US), in contrast to conventional radiography, permits visualisation of (peri)articular soft tissue structures, and compared to MRI it is relatively 

representing the changing concept of osteoarthritis (OA) in the past decade based on the introduction of new imaging techniques that permit earlier $\mathrm{OA}$ diagnosis.
Figure 1 A schema

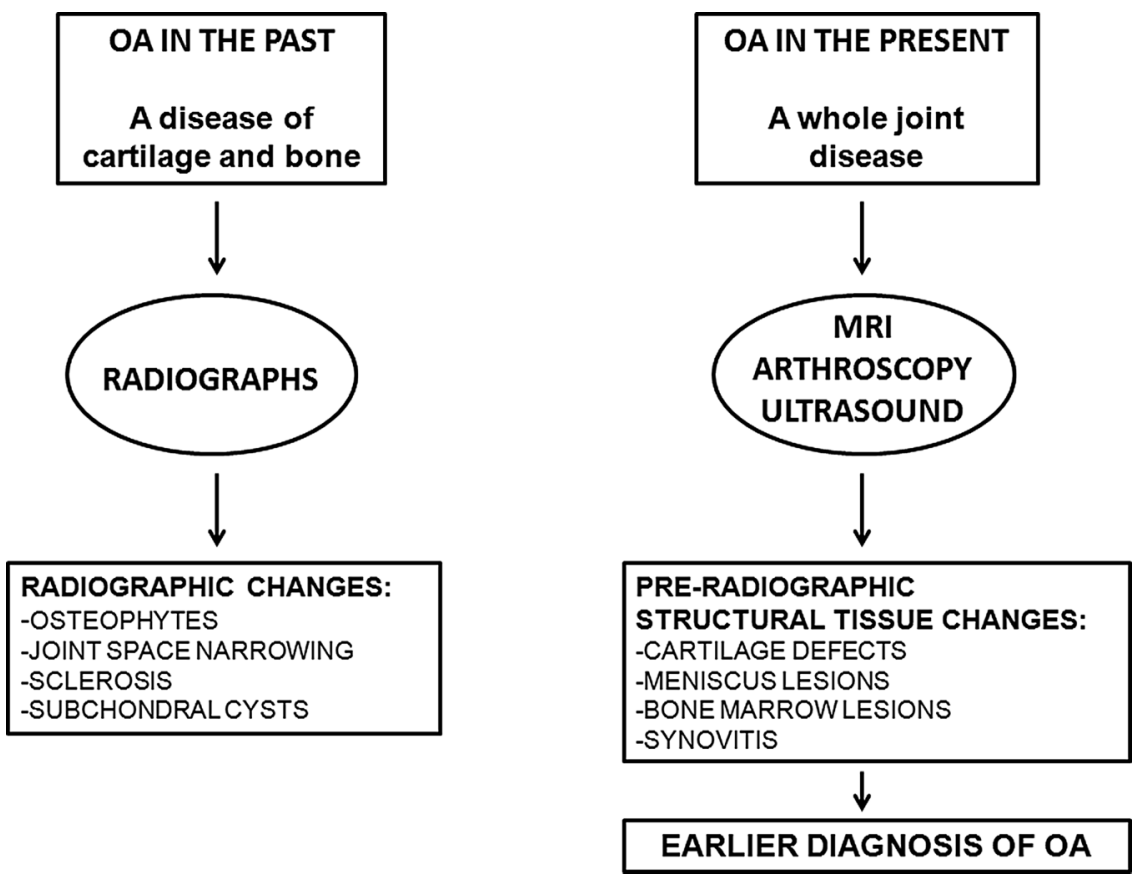

safe, inexpensive and less time-consuming. Limitations of US are its operator-dependency and its inability to assess deeper articular structures due to the acoustic shadowing. ${ }^{65}$ US can detect and evaluate both early and late abnormalities in OA involving the hyaline cartilage, synovial membrane, meniscus, joint capsule, bursa and bony cortex. Initial findings involving the hyaline cartilage are represented by blurring of the edges, which become irregular and lose the normal sharpness. Initially, they involve the superficial cartilaginous zone and correspond to micro-cleft formation attributable to tissue structural deterioration. ${ }^{66} \mathrm{OA}$ is often associated with low-grade synovitis, and synovial inflammation has been correlated with joint pain and dysfunction and, importantly, is a major risk factor for the more rapid progression of structural joint deterioration. ${ }^{67}$ US can detect synovial hypertrophy, joint effusion and increased vascularity analysed by synovial power Doppler, with a moderate to good intra-observer and interobserver reliability. ${ }^{68}$ In addition, contrast medium-enhanced (CE) musculoskeletal US has been shown to be more sensitive in detecting synovitis than CE-MRI in patients with

\section{Box 1}

Research agenda

- Identification of risk factors for developing early osteoarthritis (OA)

- Classification criteria of early OA patients

- Assessment of the role of MRI, ultrasound and other new imaging techniques in early $\mathrm{OA}$, including anatomical and functional correlations

- Assessment of standardised definitions, detection and grading of elementary lesions in early $O A$

- Evaluation of strategies to treat high-risk groups based on imaging studies suggesting early structural changes painful knee OA. ${ }^{69}$ These studies demonstrate that US is a valuable imaging tool for assessing OA joint pathology and further studies, focusing on detecting early changes of $\mathrm{OA}$, are needed with this technique.

\section{CONCLUSIONS}

The concept of OA has changed dramatically in the past decade. This is related to the development of new advanced imaging techniques, such as MRI, which can detect alterations in the structure and composition not only of cartilage and bone but also of all other joint tissues (figure 1). Not enough is known about the significance of pre-radiographic lesions such as cartilage defects, BMLs and meniscal tears detected by MRI, but it is clear that they correlate with persistent symptoms, at least in patients at high risk of developing OA. Moreover, BMLs and meniscal lesions are associated with increased incidence of cartilage defects and loss of cartilage. Cartilage defects and BMLs are potentially reversible, especially in younger participants, suggesting that intervention at early stages may forestall joint structural changes that would otherwise progress rapidly to OA. Even synovial inflammation can predict progression of OA in patients without radiographic signs. Further studies evaluating apparently healthy cohorts of patients will be necessary to permit an understanding of the significance of MRI lesions in the pathogenesis of OA. New classification criteria for EOA are needed to identify patients at high risk of OA progression in order to initiate early preventative treatments. A research agenda is tabled below (box 1).

Funding The research is supported by a grant from the Italian Ministry of Health (Ricerca Finalizzata-Giovani Ricercatori-project code: GR-2010-2317593). 
Contributors MF, SRG, MBG, RR and LP were involved in review conception and design. MF contributed in drafting of manuscript. RR, SRG, MBG and LP were involved in critical revision.

Competing interests None declared.

Provenance and peer review Commissioned; externally peer reviewed.

Data sharing statement No additional data are available.

Open Access This is an Open Access article distributed in accordance with the Creative Commons Attribution Non Commercial (CC BY-NC 4.0) license, which permits others to distribute, remix, adapt, build upon this work noncommercially, and license their derivative works on different terms, provided the original work is properly cited and the use is non-commercial. See: http:// creativecommons.org/licenses/by-nc/4.0/

\section{REFERENCES}

1. Loeser RF, Goldring SR, Scanzello CR, et al. Osteoarthritis: a disease of the joint as an organ. Arthritis Rheum 2012;64:1697-707.

2. Otero $M$, Favero $M$, Dragomir $C$, et al. Human chondrocyte cultures as models of cartilage-specific gene regulation. Methods Mol Biol 2012;806:301-36.

3. Felson DT, Hodgson R. Identifying and treating preclinical and early osteoarthritis. Rheum Dis Clin North Am 2014;40:699-710.

4. Loeser RF, Olex AL, McNulty MA, et al. Microarray analysis reveals age-related differences in gene expression during the development of osteoarthritis in mice. Arthritis Rheum 2012;64:705-17.

5. Turnbull IR, Wlzorek JJ, Osborne D, et al. Effects of age on mortality and antibiotic efficacy in cecal ligation and puncture. Shock 2003;19:310-13.

6. Goldring MB, Goldring SR. Osteoarthritis. J Cell Physiol 2007;213:626-34.

7. Madry H, Luyten FP, Facchini A. Biological aspects of early osteoarthritis. Knee Surg Sports Traumatol Arthrosc 2012;20:407-22.

8. Maroudas Al. Balance between swelling pressure and collagen tension in normal and degenerate cartilage. Nature 1976;260:808-9.

9. Saito I, Koshino T, Nakashima K, et al. Increased cellular infiltrate in inflammatory synovia of osteoarthritic knees. Osteoarthritis Cartilage 2002;10:156-62.

10. Goldring MB, Otero M. Inflammation in osteoarthritis. Curr Opin Rheumatol 2011;23:471-8.

11. Pritzker KP, Gay S, Jimenez SA, et al. Osteoarthritis cartilage histopathology: grading and staging. Osteoarthritis Cartilage 2006;14:13-29.

12. Lohmander LS, Englund PM, Dahl LL, et al. The long-term consequence of anterior cruciate ligament and meniscus injuries: osteoarthritis. Am J Sports Med 2007;35:1756-69.

13. Micheo W, Hernandez L, Seda C. Evaluation, management, rehabilitation, and prevention of anterior cruciate ligament injury: current concepts. PM R 2010;2:935-44.

14. Cimino F, Volk BS, Setter D. Anterior cruciate ligament injury: diagnosis, management, and prevention. Am Fam Physician 2010;82:917-22.

15. Brown TD, Johnston RC, Saltzman CL, et al. Posttraumatic osteoarthritis: a first estimate of incidence, prevalence, and burden of disease. J Orthop Trauma 2006;20:739-44.

16. Dare D, Rodeo S. Mechanisms of post-traumatic osteoarthritis after ACL injury. Curr Rheumatol Rep 2014;16:448.

17. Englund M, Guermazi A, Roemer FW, et al. Meniscal tear in knees without surgery and the development of radiographic osteoarthritis among middle-aged and elderly persons: the Multicenter Osteoarthritis Study. Arthritis Rheum 2009;60:831-9.

18. Englund M, Roos EM, Lohmander LS. Impact of type of meniscal tear on radiographic and symptomatic knee osteoarthritis: a sixteen-year followup of meniscectomy with matched controls. Arthritis Rheum 2003;48:2178-87.

19. Martin JA, Brown T, Heiner A, et al. Post-traumatic osteoarthritis: the role of accelerated chondrocyte senescence. Biorheology 2004;41:479-91.

20. Martin JA, McCabe D, Walter M, et al. N-acetylcysteine inhibits post-impact chondrocyte death in osteochondral explants. J Bone Joint Surg Am 2009;91:1890-7.

21. Irie $\mathrm{K}$, Uchiyama $\mathrm{E}$, Iwaso $\mathrm{H}$. Intraarticular inflammatory cytokines in acute anterior cruciate ligament injured knee. Knee 2003;10:93-6.

22. Marks PH, Donaldson ML. Inflammatory cytokine profiles associated with chondral damage in the anterior cruciate ligament-deficient knee. Arthroscopy 2005;21:1342-7.
23. Edd SN, Giori NJ, Andriacchi TP. The role of inflammation in the initiation of osteoarthritis after meniscal damage. J Biomech 2015;48:1420-6.

24. Scanzello CR, McKeon B, Swaim BH, et al. Synovial inflammation in patients undergoing arthroscopic meniscectomy: molecular characterization and relationship to symptoms. Arthritis Rheum 2011;63:391-400.

25. Ding C, Jones G, Wluka AE, et al. What can we learn about osteoarthritis by studying a healthy person against a person with early onset of disease? Curr Opin Rheumatol 2010;22:520-7.

26. Kraus VB, Birmingham J, Stabler TV, et al. Effects of intraarticular IL1-Ra for acute anterior cruciate ligament knee injury: a randomized controlled pilot trial (NCT00332254). Osteoarthritis Cartilage 2012;20:271-8.

27. Neogi T. The epidemiology and impact of pain in osteoarthritis. Osteoarthritis Cartilage 2013;21:1145-53.

28. Felson DT, Naimark A, Anderson J, et al. The prevalence of knee osteoarthritis in the elderly. The Framingham Osteoarthritis Study. Arthritis Rheum 1987;30:914-18.

29. Jordan JM, Helmick CG, Renner JB, et al. Prevalence of knee symptoms and radiographic and symptomatic knee osteoarthritis in African Americans and Caucasians: the Johnston County Osteoarthritis Project. J Rheumatol 2007;34:172-80.

30. Englund M, Guermazi A, Gale D, et al. Incidental meniscal findings on knee MRI in middle-aged and elderly persons. $N$ Engl J Med 2008;359:1108-15.

31. Yusuf E, Kortekaas MC, Watt I, et al. Do knee abnormalities visualised on MRI explain knee pain in knee osteoarthritis? A systematic review. Ann Rheum Dis 2011;70:60-7.

32. Altman R, Asch E, Bloch D, et al. Development of criteria for the classification and reporting of osteoarthritis. Classification of osteoarthritis of the knee. Diagnostic and Therapeutic Criteria Committee of the American Rheumatism Association. Arthritis Rheum 1986;29:1039-49.

33. Zhang W, Doherty M, Peat G, et al. EULAR evidence-based recommendations for the diagnosis of knee osteoarthritis. Ann Rheum Dis 2010;69:483-9.

34. Luyten FP, Denti M, Filardo G, et al. Definition and classification of early osteoarthritis of the knee. Knee Surg Sports Traumatol Arthrosc 2012;20:401-6.

35. Kellgren JH, Lawrence JS. Radiological assessment of osteo-arthrosis. Ann Rheum Dis 1957;16:494-502.

36. Schiphof D, de Klerk BM, Koes BW, et al. Good reliability, questionable validity of 25 different classification criteria of knee osteoarthritis: a systematic appraisal. J Clin Epidemiol2008;61:1205-15.

37. Felson DT, Niu J, Guermazi A, et al. Defining radiographic incidence and progression of knee osteoarthritis: suggested modifications of the Kellgren and Lawrence scale. Ann Rheum Dis 2011;70:1884-6.

38. Schiphof D, Boers M, Bierma-Zeinstra SM. Differences in descriptions of Kellgren and Lawrence grades of knee osteoarthritis. Ann Rheum Dis 2008;67:1034-6.

39. Schiphof D, de Klerk BM, Kerkhof HJ, et al. Impact of different descriptions of the Kellgren and Lawrence classification criteria on the diagnosis of knee osteoarthritis. Ann Rheum Dis 2011;70:1422-7.

40. Damen J, Schiphof D, Wolde ST, et al. Inter-observer reliability for radiographic assessment of early osteoarthritis features: the CHECK (cohort hip and cohort knee) study. Osteoarthritis Cartilage 2014;22:969-74.

41. Cicuttini FM, Wluka AE. Osteoarthritis: is OA a mechanical or systemic disease? Nat Rev Rheumatol 2014;10:515-16.

42. Roemer FW, Guermazi A. Osteoarthritis year in review 2014: imaging. Osteoarthritis Cartilage 2014;22:2003-12.

43. Peterfy CG, Guermazi A, Zaim S, et al. Whole-Organ Magnetic Resonance Imaging Score (WORMS) of the knee in osteoarthritis. Osteoarthritis Cartilage 2004;12:177-90.

44. Hunter DJ, Lo GH, Gale D, et al. The reliability of a new scoring system for knee osteoarthritis MRI and the validity of bone marrow lesion assessment: BLOKS (Boston Leeds Osteoarthritis Knee Score). Ann Rheum Dis 2008;67:206-11.

45. Mosher TJ, Walker EA, Petscavage-Thomas J, et al. Osteoarthritis year 2013 in review: imaging. Osteoarthritis Cartilage 2013;21:1425-35.

46. Hunter DJ, Zhang W, Conaghan PG, et al. Systematic review of the concurrent and predictive validity of MRI biomarkers in OA Osteoarthritis Cartilage 2011;19:557-88.

47. Ding C, Cicuttini F, Jones G. Tibial subchondral bone size and knee cartilage defects: relevance to knee osteoarthritis. Osteoarthritis Cartilage 2007;15:479-86.

48. Wang Y, Ding C, Wluka AE, et al. Factors affecting progression of knee cartilage defects in normal subjects over 2 years. Rheumatology (Oxford) 2006;45:79-84. 
49. Ding C, Cicuttini F, Scott F, et al. Natural history of knee cartilage defects and factors affecting change. Arch Intern Med 2006;166:651-8.

50. Davies-Tuck ML, Wluka AE, Wang Y, et al. The natural history of bone marrow lesions in community-based adults with no clinical knee osteoarthritis. Ann Rheum Dis 2009;68:904-8.

51. Wluka AE, Hanna F, Davies-Tuck M, et al. Bone marrow lesions predict increase in knee cartilage defects and loss of cartilage volume in middle-aged women without knee pain over 2 years. Ann Rheum Dis 2009;68:850-5.

52. Berry PA, Davies-Tuck ML, Wluka AE, et al. The natural history of bone marrow lesions in community-based middle-aged women without clinical knee osteoarthritis. Semin Arthritis Rheum 2009;39:213-17.

53. Huetink K, Stoel BC, Watt I, et al. Identification of factors associated with the development of knee osteoarthritis in a young to middle-aged cohort of patients with knee complaints. Clin Rheumatol 2014. Published Online First: 13 Sep 2014. doi:10.1007/s10067014-2774-0

54. Sharma L, Chmiel JS, Almagor O, et al. Significance of preradiographic magnetic resonance imaging lesions in persons at increased risk of knee osteoarthritis. Arthritis Rheumatol 2014;66:1811-19.

55. Guermazi A, Niu J, Hayashi D, et al. Prevalence of abnormalities in knees detected by MRI in adults without knee osteoarthritis: population based observational study (Framingham Osteoarthritis Study). BMJ 2012;345:e5339.

56. Hayes CW, Jamadar DA, Welch GW, et al. Osteoarthritis of the knee: comparison of MR imaging findings with radiographic severity measurements and pain in middle-aged women. Radiology 2005;237:998-1007.

57. Javaid MK, Lynch JA, Tolstykh I, et al. Pre-radiographic MRI findings are associated with onset of knee symptoms: the most study. Osteoarthritis Cartilage 2010;18:323-8.

58. Benito MJ, Veale DJ, FitzGerald O, et al. Synovial tissue inflammation in early and late osteoarthritis. Ann Rheum Dis 2005;64:1263-7.
59. Pearle AD, Scanzello CR, George S, et al. Elevated high-sensitivity C-reactive protein levels are associated with local inflammatory findings in patients with osteoarthritis. Osteoarthritis Cartilage 2007:15:516-23.

60. Loeser RF. Molecular mechanisms of cartilage destruction: mechanics, inflammatory mediators, and aging collide. Arthritis Rheum 2006;54:1357-60.

61. Atukorala I, Kwoh CK, Guermazi A, et al. Synovitis in knee osteoarthritis: a precursor of disease? Ann Rheum Dis 2014. Published Online First: 8 Dec 2014. doi:10.1136/annrheumdis-2014-205894

62. Roemer FW, Guermazi A, Felson DT, et al. Presence of MRI-detected joint effusion and synovitis increases the risk of cartilage loss in knees without osteoarthritis at 30-month follow-up: the MOST study. Ann Rheum Dis 2011;70:1804-9.

63. Chu $\mathrm{CR}$, Williams $\mathrm{AA}$, Coyle $\mathrm{CH}$, et al. Early diagnosis to enable early treatment of pre-osteoarthritis. Arthritis Res Ther 2012;14:212.

64. Outerbridge RE. The etiology of chondromalacia patellae. J Bone Joint Surg Br 1961;43-B:752-7.

65. Palmer AJ, Brown CP, McNally EG, et al. Non-invasive imaging of cartilage in early osteoarthritis. Bone Joint $J$ 2013;95-B:738-46.

66. lagnocco $A$. Imaging the joint in osteoarthritis: a place for ultrasound? Best Pract Res Clin Rheumatol 2010;24:27-38.

67. Scanzello CR, Goldring SR. The role of synovitis in osteoarthritis pathogenesis. Bone 2012;51:249-57.

68. Bruyn GAW, Naredo E, Damjanov N, et al. An OMERACT reliability exercise of inflammatory and structural abnormalities in patients with knee osteoarthritis using ultrasound assessment. Ann Rheum Dis 2015. Published Online First: 22 Apr 2015. doi:10.1136/ annrheumdis-2014-206774

69. Song IH, Althoff CE, Hermann KG, et al. Knee osteoarthritis. Efficacy of a new method of contrast-enhanced musculoskeletal ultrasonography in detection of synovitis in patients with knee osteoarthritis in comparison with magnetic resonance imaging. Ann Rheum Dis 2008;67:19-25. 